\title{
Metodología para los procedimientos de solución de problemas sobre Ecuaciones Diferenciales
}

\author{
Edgardo Locia \\ lociae999@hotmail.com \\ Facultad de Matemáticas \\ Universidad Autónoma de \\ Guerrero, México.
}

\author{
Otilio Mederos \\ oma8111@yahoo.es \\ Facultad de Matemáticas \\ Universidad Autónoma de \\ Coahuila, México.
}

\author{
Armando Morales \\ armando280@hotmail.com \\ Facultad de Matemáticas \\ Universidad Autónoma de \\ Guerrero, México.
}

José M. Rodríguez

jomaro@math.uc3m.es

Dpto de Matemáticas

Universidad Carlos III. Madrid, España
José M. Sigarreta

josemariasigarretaalmira@hotmail.com

Facultad de Matemáticas

Universidad Autónoma de

Guerrero, México.

Recibido: Marzo 13, 2013

Aceptado: Noviembre 12, 2013

\begin{abstract}
Resumen. En este trabajo, se propone una metodología para favorecer la enseñanza y aprendizaje de los procedimientos de solución de problemas sobre ecuaciones diferenciales, a nivel universitario. Los elementos teóricos y metodológicos se basan en el proceso de resolución de problemas desarrollado a partir del enfoque histórico cultural de Vigotsky y Teoría de la Actividad de Leontiev. En la propuesta se resaltan dos aspectos fundamentales que garantizan su efectividad en la práctica docente, por una parte, se propone una clasificación y caracterización de los problemas matemáticos (problemas estructurales y problemas reductivos). Por otra parte, a partir de la caracterización desarrollada, se estructura una estrategia de solución basada en las tres fases fundamentales asociadas con la Teoría de la Actividad: orientación, ejecución y control, las cuales permiten evaluar el aprendizaje a lo largo de todo el proceso.
\end{abstract}

Palabras clave: Ecuaciones diferenciales, problemas, estrategia heurística, metodología, enseñanza 
Abstract. In this paper, we propose a methodology for problem solving procedures on differential equations, at the university level. The theoretical and methodological elements are based on problem solving process from Vygotsky's cultural historical approach and activity theory developed by Leontiev. Two essential elements that guarantee its effectiveness in teaching are highlighted. On one hand, we propose a classification and characterization of mathematical problems (structural and reductive). On the other hand, shows a strategy for problem solving based on the phases of activity theory, which allow to evaluate the process.

KeyWords: Differential equations, heuristic strategy, methodology, teaching

\subsection{Introducción}

Existen investigaciones como las que señalan (Polya, 1981; Labarrere, 1988; Rodríguez, 1991; Schoenfeld, 1991; Campistrous y Rizo, 1996; Sigarreta et al, 2001-2009) que avalan la importancia de la resolución de problemas como uno de los aspectos fundamentales a los que debe estar orientado el trabajo del profesor en el proceso enseñanza-aprendizaje.

Por otro lado, no es difícil constatar que el tratamiento de las ecuaciones diferenciales en algunas escuelas de nivel superior, generalmente se limita a la enseñanza de procedimientos sin darle significado a los conceptos, como se evidencia en diversos trabajos de investigación (por ejemplo, Rodríguez (1991) y Sigarreta et al $(2008,2009))$. En estos trabajos se hace evidente que, aunado a la creencia arcana de que las matemáticas se pueden enseñar con sólo tener el dominio profundo del contenido, la utilización de libros de texto, de manera mecánica y directa, se hace cómplice de la generación de deficiencias en el alumnado, llegando al punto de confundir la resolución de problemas como un fin en sí mismo y soslayando la forma en que se consigue un determinado resultado. Es necesario transformar este modo arcaico de enseñanza a uno basado en el descubrimiento, el pensamiento creativo y original, sustentado en la resolución de problemas como medio privilegiado de generación de saber y no como un fin desarticulado y vacío de significados.

En función de lo planteado anteriormente, en este artículo presentamos una estrategia metodológica para favorecer los procesos de solución de problemas relacionados con ecuaciones diferenciales en el nivel superior, que utilice las potencialidades de la heurística en el proceso de enseñanza-aprendizaje.

\subsection{Elementos teórico - metodológicos.}

\subsection{Caracterización del concepto problema.}

Para el análisis del significado del término "problema", partamos de su uso en el léxico común; en su más amplia representación se utiliza para exponer una situación, de la cual se busca un resultado a partir de ciertos datos. En el diccionario Aristos (1980), está asentado: Cuestión o proposición dudosa que 
se trata de resolver. Proposición encaminada a averiguar el modo de obtener un resultado cuando se conocen ciertos datos. Mientras que en el diccionario Cervantes (1978), encontramos: Cuestión que se trata de resolver por procedimientos científicos, Mat: proposición dirigida a averiguar el modo de obtener un resultado.

Sin embargo, cuando se habla de problemas, para los dedicados a la enseñanza de las matemáticas, su significado se extiende; por tanto, si se pretende realizar un análisis profundo de la definición de problema, hay que investigar la dimensión psicopedagógica y particularizarlo desde el punto de vista de la Didáctica de la Matemática. Se hará el análisis basado en las palabras de Hadamard (1945) cuando expresó:

"... este asunto envuelve dos disciplinas, Psicología y Matemática, y requerirá ser tratado adecuadamente en ese orden, por ambos, tanto por el psicólogo como por el matemático. Por la falta de esta composición, el asunto ha sido investigado por los matemáticos por un lado y por los psicólogos por el otro..." (Hadamard, J. 1945, p. 1).

Desde los postulados de la Psicología se estudiaron dos definiciones trascendentales en este campo, las dadas por Rubinstein (1966) y Leontiev (1986). Del análisis de las definiciones establecidas por estos psicólogos encarnan dos características comunes: en todo verdadero problema el sujeto desconoce la vía de solución y al posicionarse frente al problema mismo adopta un carácter activo. Para Majmutov (1983) los elementos fundamentales de un problema son lo conocido y lo desconocido (hallar el nexo, las relaciones entre lo conocido y lo desconocido).

Borasi (1986), relaciona problemas con texto a los textos formulados con precisión, donde aparecen todos los datos necesarios para obtener la solución. También trabaja los problemas para el entretenimiento y las pruebas de conjeturas, refiriéndose a la demostración de teoremas o de una cierta propiedad. Uno de los inconvenientes es que no queda clara la base para la división de los mismos.

Para Kantowski (1981) un problema "es una situación que difiere de un ejercicio en que el resolutor de problemas no tiene un proceso algorítmico que lo conducirá con certeza a la solución." (Kantowski, M. 1981, p. 111). Otra definición que aparece como constante en un conjunto de investigaciones sobre el campo de la resolución de problemas, es la dada por Palacios y Zambrano (1993) que precisa: "El problema puede ser definido como cualquier situación, que produce por un lado un cierto grado de incertidumbre y, por otro lado, una conducta tendente a la búsqueda de su solución". (Palacios, C. y Zambrano, E. 1993, p. 52). Aunque en la definición anterior se observa una cierta relación en el significado que se le atribuye a los términos utilizados, se entiende que la dada por estos autores es más acabada que las precedentes, pues plantea de una manera más directa los elementos esenciales de la definición. En esa misma dirección Campistrous y Rizo (1996), definen problema como "toda situación en la que hay un planteamiento inicial y una exigencia que obliga a transformarla. La vía de pasar de la situación o planteamiento inicial a la nueva situación exigida tiene que ser desconocida y la persona debe querer realizar la transformación" (Campistrous, L. y Rizo, C. 1996, p. IX).

$\mathrm{Al}$ analizar estas dos últimas definiciones encontramos elementos que son de suma importancia para hacer una caracterización del concepto de problema escolar, lo que permite un acceso lingüísticoconceptual de mayor precisión en la elaboración de los problemas y que los profesores reconozcan cuándo están realmente en presencia de ellos. Estos elementos son: a) la vía de pasar de la situación inicial a la nueva situación debe ser desconocida; estableciendo diferencias esenciales entre ejercicio y problema; b) la persona quiere realizar esa transformación, teniendo bien en claro que lo que constituye un problema para uno puede no serlo para otro. 
Dicho análisis puede resumirse en el hecho de que, aunque existe una gran diversidad de criterios, los autores de manera general no se contradicen; en tal sentido, los rasgos fundamentales analizados son:

- Existirá una situación inicial o varias situaciones iniciales y una o varias situaciones finales

- La vía de pasar de la situación inicial o situaciones iniciales a la situación final o situaciones finales debe de ser desconocida o que no se pueda acceder a ellas de forma inmediata

- Debe existir la persona que quiera resolverla

- La persona dispone de los elementos necesarios para buscar las relaciones que le permitan transformar la situación o situaciones planteadas

\subsection{Clasificaciones de los problemas en su relación con las ecuaciones diferenciales.}

Todos los autores parecen estar de acuerdo en que un elemento fundamental para dirigir el proceso de enseñanza-aprendizaje de la Matemática, es que tanto el profesor como el estudiante sepan en presencia de qué clase de problemas se encuentran; en tal dirección han aparecido en los últimos años muchos tipos de clasificaciones. Sobre la base del contenido semántico de problema, Majmutov (1983) plantea que los problemas se pueden clasificar de acuerdo con diferentes bases. Por ejemplo, partiendo de la significación del término "problema", como cuestión que debe ser realizada, y como interrogante que debe ser resuelta. Los problemas, según este autor, por su contenido, es decir, en función de la información que transmite el texto o planteamiento del problema, se pueden dividir en: cotidianos, jurídicos, pedagógicos, entre otros. Es claro que esta clasificación es poco práctica desde el punto de vista didáctico, porque las fronteras entre unos y otros problemas se solapan.

Para adentrarnos en el estudio de las tipologías, es menester plantear que los pasos iniciales, desde el punto de vista docente, giraban en torno a la naturaleza de la asignatura. Esta clasificación es la más generalizada dentro de los profesores de Matemática en la actualidad, aunque se confirma que existen problemas de Matemática, Física, Química, etcétera, y otros que no estén dentro de ninguna asignatura, que son los de razonamiento lógico.

Ya en los trabajos de Polya (1945) se diferencia los problemas por resolver y los problemas por demostrar; por su parte González (1954) los distingue en particulares y generales. Mientras que en los trabajos de Bertoglia (1990) aparece una clasificación que está más acabada, ya que se enfatiza no sólo en el proceso de solución, sino que además pone al descubierto la utilización de la lógica dentro del proceso; su clasificación se centra en dos grandes grupos llamados: Problemas Cerrados. En estos, la solución se deduce de forma lógica a partir de la información que aparece en el planteamiento del problema y que resulta suficiente para encontrar la respuesta correca. Problemas Abiertos. En estos, el resolutor necesita ir más allá de la información recibida, utilizándola de manera directa y/o modificando los significados atribuidos a los elementos del problema.

Al no analizarse las ecuaciones diferenciales como objeto de la resolución de problemas, sucede que dentro de las clasificaciones estudiadas, no aparecen las tipologías en esta dirección. Para el logro de los objetivos de la enseñanza-aprendizaje de la matemática, en particular los relacionados con la resolución de problemas, hay que tener presente que los contenidos a tratar deben verse en dos direcciones: como objeto de apropiación (aprender ecuaciones diferenciales) y como base para el desarrollo de la resolución de problemas. Los educadores matemáticos han puesto al descubierto que la resolución de problemas debe ser el objetivo instructivo fundamental en la enseñanza de las matemáticas, aunque no 
la única habilidad a desarrollar.

Las clasificaciones de problemas en la dirección antes señalada no están dadas de forma explícita, elemento examinado anteriormente, pero es prudente aclarar que ya en los trabajos de Zillmer (1981), se dan pautas para tratar determinados problemas con el objetivo de desarrollar diferentes aspectos relacionados con la resolución de problemas.

Las ideas desarrolladas sirvieron de base o sustento para clasificar los diferentes tipos de ecuaciones diferenciales y delimitar sus características. Esta clasificación encierra dos grandes grupos de problemas con sus respectivos subgrupos y entre ellos forman un sistema para el desarrollo de procedimientos de solución de las ecuaciones diferenciales. Con esta posición se tienen en cuenta tres aspectos que son indispensables al concebir un sistema: elementos que lo integran, relación que se establece entre los elementos del sistema y resultado que se genera de esta relación.

El objetivo de la clasificación está dado, en lo fundamental, en organizar la actividad y determinar el efecto que provoca el sistema de problemas en el desarrollo de un determinado procedimiento de solución y viceversa. Esta división permitirá revelar la estructura interna de los problemas y, además, ayudará a los profesores a estructurarlos en función de cada uno de los elementos a desarrollar, de forma tal que se alcance un proceso óptimo de enseñanza-aprendizaje en la resolución de problemas para favorecer el proceso de enseñanza aprendizaje de las ecuaciones diferenciales.

Así pues, la clasificación que se propone está encaminada al procedimiento que se quiera utilizar, además debe contener en su totalidad todas las posibles situaciones problema que se puedan presentar en un curso de ecuaciones diferenciales. La clasificación que proponemos es la siguiente:

1. Problemas estructurales: Son aquellos cuya similitud de estructura y forma, así como de solución nos permiten aplicar alguna de las analogías siguientes:

a) Analogía de estructura: Son aquellos problemas donde se evidencia similitud de cuerpo y forma, y al aplicar una analogía podemos llevar nuestros problemas a situaciones donde se pueda evidenciar una solución en base a un problema que ya se conoce.

b) Analogía de solución: En este rubro, si un problema tiene semejanzas notables en su proceso de solución entonces se estaría en posición de elaborar una estrategia que lleve a la solución deseada.

2. Problemas Reductivos: Son aquellos que tienen solución en problemas posiblemente de distinto carácter o dimensión. En donde aplicamos los siguientes principios:

a) Principio de Transformación: Aquellos problemas que se pueden llevar a otra dimensión mediante la transformación de alguno o todos sus componentes, teniendo la seguridad de que dándole respuesta al nuevo problema se le dará también al anterior.

b) Principio de Reducción: Son aquellos problemas que se llevan a un rubro distinto donde al hacer una aplicación recursiva o inmediata se le encontrará la solución al problema original.

Las características de los problemas por analogía estructural utilizados son:

Problemas con datos insuficientes, donde el estudiante se ve obligado a buscar problemas semejantes donde se evidencie cuál es el dato faltante. 
Problemas con datos superfluos, aquí el estudiante tendrá que apreciar otros problemas para aclarar los datos que le son dados.

Problemas donde aparezcan datos contradictorios, de igual forma, al no poder encontrar vías de solución optará por buscar problemas semejantes de cuerpo y forma, y así emitir juicios de valor sobre los datos del problema. Incluso podría analizar cuáles deberán ser las características de los datos para que pueda tener una solución.

Presentación de soluciones de problemas con errores "sutiles" o difíciles de detectar a priori, esto llevará eventualmente al estudiante a echar vistazos a ejercicios similares.

Las características de los problemas por analogía de solución utilizados son:

Problemas en los que, dado un conjunto de premisas, se pida obtener un conjunto de tesis, si el estudiante necesita realizar conclusiones de un problema al hacer otro puede aplicar el mismo proceso que utilizó para resolver el primero.

Presentación de soluciones de problemas, con una vía de solución correcta y otra incorrecta, el analizar qué procedimientos pueden llevar o no a la solución del problema creará una base de dichos procedimientos para su futura implementación en diversos ejercicios.

Presentación de soluciones diferentes a un mismo problema, el presentar diversas soluciones atraerá al estudiante a identificar procedimientos más sencillos o más complicados y así decidir su debida aplicación en otros problemas.

Las características de los problemas por principio de transformación utilizados son:

Problemas que no exijan cálculo para encontrar su solución, pero requieran de una sólida fundamentación teórica, el tener una fundamentación teórica de los datos necesarios para resolver el problema llevará al estudiante a buscar su solución en situaciones de equivalencia.

Problemas cuya respuesta requiera un análisis complementario, el tener que efectuar análisis de los datos buscados y los encontrados evidenciará las relaciones que existen entre los datos y aquellos que se utilizaron para su solución, así pues, obtendrá un marco de conocimientos que se pueden utilizar para la solución de otros problemas.

Las características de los problemas por principio de reducción utilizados son:

Problemas cuya resolución requiera de la resolución de sub-problemas, el usar sub-problemas como solución del original es claramente la aplicación de una reducción del problema a otros ya conocidos.

Problemas que exigen para su solución la búqueda de una información que él no posee, el buscar información que no se tiene de facto puede llevar a encontrar la solución en la reducción a conocimientos nuevos, o a conocimientos que no eran evidentes en un principio.

Problemas que, dado el nivel de conocimiento del estudiante, le sea cómodo obtener múltiples vías de soluciones, el obtener diferentes vías de soluciones le dará al estudiante la pauta para llevar a cabo 
procesos de reducción, que eventualmente buscará con naturalidad en otros problemas.

A modo de ejemplo veamos algunos problemas con las características anteriores:

1. Estamos aprendiendo problemas de analogía de estructura en el tema de ecuaciones homogéneas, para lo cual ya se ha resuelto el problema $x y^{\prime}=\sqrt{x^{2}-y^{2}}+y$. Después se aborda el problema $\left(x y^{\prime}-y\right)^{2}-x^{2}=y^{2}-2 x^{2}$. Si el estudiante logra encontrar una estructura similar al ejercicio anterior intentará utilizarlo para resolver este nuevo problema. Este problema estaría enmarcado en los datos superfluos, ya que hay una cantidad de datos que no se verían relacionados con los problemas anteriores y el aplicar directamente el método de solución que se está enseñando sería poco redituable. La solución del primer problema es de la siguiente manera:

$$
x y^{\prime}=\sqrt{x^{2}-y^{2}}+y
$$

Hacemos $y=u x$, entonces $y^{\prime}=u^{\prime} x+u$. Sustituimos en la ecuación original $y$ e $y^{\prime}$.

$$
\begin{gathered}
x\left(u^{\prime} x+u\right)=\sqrt{x^{2}-(u x)^{2}}+u x \\
u^{\prime} x^{2}+u x=x \sqrt{1-u^{2}}+u x \\
\frac{d u}{d x} x=\sqrt{1-u^{2}} \\
\frac{d u}{\sqrt{1-u^{2}}}=\frac{d x}{x}
\end{gathered}
$$

Entonces $\operatorname{arcsen} u=\ln |C x|$.

Sustituimos $u=\frac{y}{x}, \mathrm{y}$ nos queda arcsen $\frac{y}{x}=\ln |C x|$, por lo tanto

$$
y=x \operatorname{sen}(\ln |C x|)
$$

para valores de $x$ distintos de cero.

En el nuevo problema, tratamos de encontrar una estructura similar a la del problema anterior haciendo lo siguiente:

$$
\begin{gathered}
\left(x y^{\prime}-y\right)^{2}-x^{2}=y^{2}-2 x^{2} \\
\left(x y^{\prime}-y\right)^{2}=y^{2}-x^{2} \\
x y^{\prime}-y=\sqrt{y^{2}-x^{2}}
\end{gathered}
$$

en este caso, se consideró el signo positivo para poder establecer la analogía. Entonces,

$$
x y^{\prime}=\sqrt{y^{2}-x^{2}}+y .
$$

Observamos entonces que el nuevo problema tiene semejanza de estructura y podemos resolverlo como se resolvió el anterior. Nótese que se está aplicando un recurso heurístico de reducción a un 
problema ya conocido, sin embargo, la búsqueda está dirigida hacia uno que tenga semejanza de estructura. Aquí la ganancia principal no es la utilización de un método para resolver ecuaciones, sino la búsqueda de formas para resolverlos.

2. Un problema por analogía de solución podría ser: resolver la siguiente ecuación diferencial

$$
x y^{\prime}+y=x^{2} \operatorname{sen} x ; \text { o bien } y^{\prime}+\frac{y}{x}=x \operatorname{sen} x, x \neq 0 .
$$

Hacemos $y=u(x) v(x)$, entonces $y^{\prime}=u^{\prime}(x) v(x)+u(x) v^{\prime}(x)$. Y sustituimos en la ecuación: $u^{\prime} v+$ $u v^{\prime}+\frac{u v}{x}=x \operatorname{sen} x$. Al factorizar $v$ nos queda la ecuación:

$$
v\left(u^{\prime}+\frac{u}{x}\right)+u v^{\prime}=x \operatorname{sen} x
$$

Buscamos la solución particular para $u^{\prime}+\frac{u}{x}=0 ; \quad \frac{d u}{d x}=-\frac{u}{x} ; u=\frac{1}{x}$.

Sustituimos $u$ en el resto de la ecuación:

$$
u v^{\prime}=x \operatorname{sen} x ; \frac{1}{x} v^{\prime}=x \operatorname{sen} x ; v^{\prime}=x^{2} \operatorname{sen} x ; d v=x^{2} \operatorname{sen} x d x
$$

Con esto tenemos $v=-x^{2} \cos x+2 x \operatorname{sen} x+2 \cos x+C$. Finalmente tenemos $y=u v=\frac{1}{x}\left(-x^{2} \cos x+\right.$ $2 x \operatorname{sen} x+2 \cos x+C)$ o bien $y=-x \cos x+2 \operatorname{sen} x+\frac{2}{x} \cos x+\frac{C}{x}$.

Es claro que resolver un problema desde distintos ángulos, permite analizar con mayor profundidad el método de solución empleado. Nótese que para la solución del problema anterior, la idea central radica en suponer como solución una función expresada como el producto de dos funciones, para poder utilizar la conocida relación asociada a la derivada del producto de funciones. En esa misma dirección veamos la siguiente solución:

$$
\begin{gathered}
x y^{\prime}+y=x^{2} \operatorname{sen} x \\
\frac{d}{d x}(x y)=x^{2} \operatorname{sen} x \\
d(x y)=x^{2} \operatorname{sen} x d x \\
x y=-x^{2} \cos x+2 x \operatorname{sen} x+2 \cos x+c \\
y=-x \cos x+2 \operatorname{sen} x+\frac{2}{x} \cos x+\frac{c}{x}
\end{gathered}
$$

3. Un problema reductivo: por principio de transformación. Resolver la siguiente ecuación diferencial $y=x y^{\prime}+\frac{a}{y^{\prime 2}}$.

Hacemos $y^{\prime}=p$. Entonces $y=x p+\frac{a}{p^{2}}$, derivando ambas partes de la igualdad dada respecto de $x$ se tiene que: $y^{\prime}=x \frac{d p}{d x}+p-\frac{2 a}{p^{3}} \frac{d p}{d x}$. Como $y^{\prime}=p$, entonces $p=x \frac{d p}{d x}+p-\frac{2 a}{p^{3}} \frac{d p}{d x}$, luego; 
$\frac{d p}{d x}\left(x-\frac{2 a}{p^{3}}\right)=0$

Resolviendo la ecuación $x-\frac{2 a}{p^{3}}=0$ se tiene $x=\frac{2 a}{p^{3}}$. Al sustituir el resultado en la ecuación original se obtiene $y=\left(\frac{2 a}{p^{3}}\right) p+\frac{a}{p^{2}}$. Iremos desarrollando la ecuación hasta el punto en que podamos sustituir el valor de $p$, en función de $x: y=\frac{2 a}{p^{2}}+\frac{a}{p^{2}}=\frac{3 a}{p^{2}}$.

De la ecuación $x=\frac{2 a}{p^{3}}$ tenemos que $p^{3}=\frac{2 a}{x}$. La ecuación $y=\frac{3 a}{p^{2}}$ la elevamos al cubo y se obtiene: $y^{3}=\frac{27 a^{3}}{p^{6}}$

Al sustituir $p^{3}=\frac{2 a}{x}$ en la ecuación anterior obtenemos $y^{3}=\frac{27 a^{3}}{\left(\frac{2 a}{x}\right)^{2}}$.

Al hacer la simplificación se obtiene $4 y^{3}=27 a x^{2}$ que es la solución implícita de la ecuación dada.

Resulta atinado plantear que el sistema de problemas, cualesquiera sean las características que posea para incidir en cualquier dimensión, debe estar bien organizado y permitirle al estudiante tener, en un primer nivel, posibilidades de éxito. La utilización del contenido de la información que se trate en el texto del problema es de suma importancia; pensamos que en este sentido la presentación y estructura del problema son las vías fundamentales para lograr el entendimiento de los procesos heurísticos implicados en la solución de los problemas.

\subsection{Estrategia para la resolución de problemas.}

Los elementos anteriores sustentan el proceso de resolución de problemas, pero al ser un fenómeno tan complejo se necesita un modelo que permita guiar la actividad que en tal sentido realiza el estudiante. Esta necesidad puntualiza la importancia de profundizar en el estudio de las estrategias de solución de problemas, tanto generales como específicas, como vía para favorecer el proceso de resolución de problemas y aprovechar sus potencialidades para dar una instrucción heurística así como la mejora sustancial de los procesos metacognitivos de los estudiantes.

Si se pretende lograr resultados significativos es preciso enseñar a los estudiantes estrategias generales y técnicas de trabajo que permitan ganar seguridad en él mismo, confiando en que las habilidades que posee son suficientes para abordar el problema.

Son varios los autores dedicados a la búsqueda y obtención de estrategias heurísticas para la resolución de problemas. Ratificando la posición tomada, en el trabajo se considera que no es la falta de estrategias la causa fundamental por la que los profesores presentan dificultades con la enseñanza de la resolución de problemas y los estudiantes con el aprendizaje. Las dificultades principales recaen en las operaciones propuestas en cada acción del proceso, en cómo se enseñan (profesor) y en cómo se usan (estudiantes). Un peso fundamental recae en las operaciones a realizar, al constituir las mismas el comportamiento general en la búsqueda de la solución de problemas y el elemento que permite 
vincular el problema con la esfera afectiva del estudiante y con el medio en que se desarrolla.

Aunque existen múltiples estrategias para la resolución de problemas, por lo general los estudiantes no trazan un plan de trabajo, no analizan exhaustivamente los datos de que disponen ni tratan de relacionar estos datos y la incógnita con conocimientos previos que enlacen ambas cuestiones. Tampoco son capaces, en muchas ocasiones, de clasificar el problema a trabajar o de realizar un cambio en el dominio en el que se presenta el problema para desarrollarlo en un dominio más sencillo, operar en éste y posteriormente regresar al dominio original. En tal dirección, en este trabajo vemos la resolución de problemas como un elemento dentro de la teoría de la actividad desarrollada por Leontiev (1976), en la que se que se proponen tres etapas fundamentales para la realización de las acciones: orientación, ejecución y control.

Las indicaciones para la solución de problemas son variadas según el tipo de problema a resolver. Por ejemplo, si el problema requerido relacionado con las ecuaciones diferenciales tiene carácter cuantitativo, es preciso superar la tendencia común a encontrar lo más pronto posible un dato, que a menudo no se sabe interpretar y del que se pueden obtener conclusiones absurdas. Es preciso ayudar a los alumnos a diferenciar el problema científico del matemático, haciendo especial hincapié en la reflexión cualitativa, retrasando lo más posible su cuantificación. Los problemas cualitativos en este tema suelen tener dificultades de comprensión conceptual, por lo que es preciso establecer relaciones significativas con los conocimientos previos, Lee (2009).

La reflexión sobre el proceso de aprendizaje y la evaluación de resultados supone hacer conscientes los procesos mentales que se han utilizado, así como el uso de los conocimientos que se han movilizado y la evolución que han seguido a través del proceso de aprendizaje. Ello permite una interacción con el profesor y los estudiantes y destacar aquellas estrategias que resultaron más adecuadas. La reflexión continúa sobre las estrategias que se van usando ante la resolución de un problema; lo cual es un proceso imprescindible para adquirir habilidades mentales duraderas, que pueden transferirse a la solución de nuevas interrogantes.

Como se mostró en los párrafos anteriores, se considera a la resolución de problemas relacionados con las ecuaciones diferenciales como un elemento dentro de la teoría de la actividad desarrollada por Leontiev (1976). Cada acción está dirigida por un conjunto de operaciones que se deberán realizar en un principio con la ayuda del profesor, y eventualmente por el estudiante mismo. Las acciones están enmarcadas en las tres acciones de dicha teoría: Orientación, Ejecución y Control. La acción 1 está inmersa en la Orientación, la acción 2 en la Ejecución, y la acción 3 en la de control.

Acción 1: Orientación. Operaciones: ¿Qué problema vas a enfrentar? ¿Has visto alguno formulado de manera parecida? ¿Es un problema real? ¿Está relacionado con tu entorno sociocultural? ¿Qué relaciones reales son expresadas en el texto del problema? ¿Qué elementos conoces sobre la actividad abordada en el texto del problema? ¿Son familiares para ti todos los términos que intervienen en la formulación del problema? ¿En qué campo de conocimientos se mueve el problema planteado?

Acción 2: Ejecución. Operaciones: Clasifica el problema: estructurales o reductivos, en su debida subclasificación. Elabora un esquema, diagrama, tabla, etc. ¿Son suficientes los datos? ¿Existen datos contradictorios? Delimita qué conocimientos se relacionan con los elementos del problema. Realiza transformaciones equivalentes en la premisa y/o la tesis. ¿Has resuelto un problema parecido o relacionado con éste?. Escoge un lenguaje apropiado o una notación adecuada. 
Acción 3: Control. Operaciones: ¿Todas las soluciones halladas son soluciones del problema? Explica con tus palabras cómo arribaste a la solución. ¿Puede ser generalizado el método de solución encontrado?

La primera acción de la actividad aparece como elemento esencial para el logro del objetivo, ya que sirve de elemento inductor, es decir, refuerza los atributos que dentro del problema permiten la aparición del motivo de la actividad. Además, el conjunto de preguntas propuesto crea las bases para la comprensión del fin de la actividad, impulsando con ello la aspiración de alcanzar tal fin. Las operaciones a realizar favorecen el uso de sus experiencias en la propia práctica social como elemento para la solución del problema en cuestión. Un elemento imprescindible a tener en cuenta en la primera acción es que el surgimiento de un problema no aparece de manera directa con la presentación formal del mismo, sino que aparece después de ser interiorizado por el estudiante y emerge de manera más rápida o directa si está relacionado con el contexto sociocultural en el cual se desarrolla el estudiante, que son los elementos puntualizados en las operaciones que se proponen.

La segunda acción se relaciona con el componente afectivo-volitivo al contemplar los medios adecuados para lograr el fin, la valoración de los posibles procedimientos a seguir, entre los que se debe tomar una posición de toma de decisión al elegir uno de ellos; y a su vez permiten analizar, además, las ventajas o desventajas de un determinado camino. El profesor debe provocar no sólo la reflexión de los componentes externos con que se cuenta para la realización de la actividad, sino los posibles obstáculos internos, creando en los estudiantes una cierta confianza en sus posibilidades. Asimismo, estas acciones permiten crear un modo de actuación que sienta las bases para el análisis crítico del problema matemático y la valoración de situaciones de conflictos.

La acción número 3 aparece porque la resolución de problemas matemáticos necesita elevarse al plano teórico para potenciar las generalizaciones, a partir del resultado obtenido en la actividad realizada. Además, su importancia está dada en que el hombre, como ser consciente, siempre verifica la satisfacción del motivo que lo impulsó a realizar la actividad y esta verificación no es más que la acción de control, y, aunque no aparezca declarada, se realiza en todo momento del desarrollo de la estrategia. Las operaciones propuestas para esta acción tributan, en primer lugar, a la valoración, al analizar el proceso seguido para su solución de manera general, además de relacionarse cada uno de los componentes de la heurística analizados.

A título de ejemplo de la aplicación de la estrategia heurística se muestran los siguientes ejemplos:

\section{Ejemplo 1.1}

Demostrar que la curva que posee la propiedad de que todas sus normales pasan por un punto constante es una circunferencia.

(Acción 1).

- Nos preguntamos cuáles son las expresiones de mayor valor semántico: curva, normal, punto constante, circunferencia.

- Buscamos sinónimos de algunas expresiones: Normal-recta perpendicular a la tangente. 
- Establecemos la incógnita: Una función $f(x)$ que cumpla determinadas propiedades.

- Enunciamos el problema con nuestras propias palabras. Buscamos encontrar un procedimiento irrefutable que haga ver que la curva es una circunferencia.

(Acción 2).

- Elaboramos un esquema que describa lo mejor posible el problema:

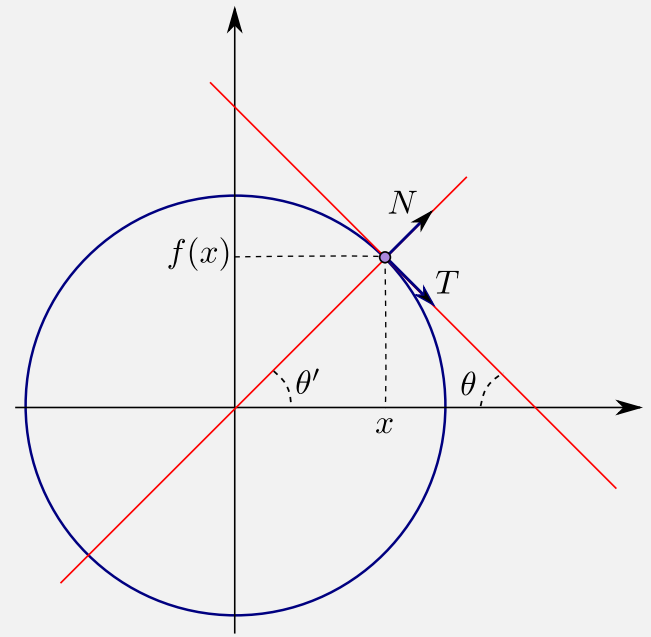

Figura 1.1

- Parece que los datos son suficientes.

- Ya que pudimos elaborar un esquema, parece que no hay datos contradictorios.

- Tampoco parece haber datos sobrantes.

- Relacionamos los datos con la incógnita: relacionamos la recta normal con la recta tangente, y el punto constante con el punto $(0,0)$.

- Transformamos el problema en uno equivalente: el problema lo transformamos en resolver una ecuación diferencial, que hipotéticamente deberá ser la ecuación de una circunferencia.

- El campo de conocimiento donde lo ubicamos es el de la geometría.

- Los conocimientos que se relacionan con los elementos del problema son curva, ecuación diferencial, tangente a una curva, normal.

- Recordamos si ya hemos resuelto algún problema parecido o relacionado, si es así, nos damos a la tarea de crear una estrategia de solución parecida para este problema.

- Al parecer nuestro diagrama es una representación general de las posibles situaciones, y entonces procedemos con determinación a establecer una relación de los conocimientos que necesitamos y los datos que tenemos, y así buscar una solución. 
- A partir de la gráfica que hemos hecho, podemos decir que: Del triangulo rectángulo formado por el punto $(0,0),(x, f(x)),(x, 0)$ :

$$
\tan \theta^{\prime}=\frac{f(x)}{x}
$$

Además, por la definición de derivada de una función tenemos que:

$$
\tan \theta=f^{\prime}(x)
$$

Aquí es importante mirar cuál es la relación entre las dos tangentes anteriores, ¿Cuál es la relación entre las tangentes de 2 rectas perpendiculares? Si el estudiante sabe la relación las cosas fluyen, sin embargo, se puede invitar al estudiante a buscar esa información. Entonces la relación es:

$$
\begin{aligned}
& \tan \theta \tan \theta^{\prime}=-1 \\
& \tan \theta^{\prime}=-\frac{1}{\tan \theta}
\end{aligned}
$$

Así obtenemos la siguiente ecuación:

$$
-\frac{1}{f^{\prime}(x)}=\frac{f(x)}{x}
$$

La cual es una ecuación diferencial que trataremos de resolver.

$$
\begin{gathered}
-x d x=y d y \\
-\frac{x^{2}}{2}+C=\frac{y^{2}}{2} \\
-x^{2}+C=y^{2} \\
x^{2}+y^{2}=C
\end{gathered}
$$

Esto es evidentemente una ecuación de una circunferencia del radio que se quiera $\sqrt{C}$.

(Acción 3).

- Escogemos un lenguaje apropiado para expresar la solución: La curva que tiene la propiedad que sus normales pasan por un punto constante es un punto constante, efectivamente es una circunferencia.

- Nos preguntamos si puede ser generalizado el método de solución. Al haber aplicado un método similar al de un problema anterior, la generalización de este proceso para problemas similares está cobrando fuerza.

- ¿Responde realmente al problema? Sí, porque a partir de la información dada dedujimos una ecuación que cumple las propiedades y que además, efectivamente es una circunferencia. 


\section{Ejemplo 1.2}

Resuelve la siguiente ecuación

$$
y^{\prime \prime}+2 y^{\prime}+y=0
$$

(Acción 1).

- Nos preguntamos cuáles son las expresiones de mayor valor semántico: función, ecuación.

- Establecemos la incógnita: Una función $f(x)$ que cumpla determinadas propiedades.

- Enunciamos el problema con nuestras propias palabras: buscamos una función derivable respecto de $x$ que cumpla la propiedad que $y^{\prime \prime}+2 y^{\prime}+y=0$.

- Parece que los datos son suficientes.

- Tampoco parece haber datos sobrantes.

- El campo de conocimiento donde lo ubicamos es el del análisis.

- Lo clasificamos: le damos la clasificación de estructural por analogía de estructura.

(Acción 2).

- A partir de recordar si se han resuelto algunos problemas parecidos o relacionados al dado y en base a la estructura, se propone la tarea de crear una estrategia de solución.

- De la expresión literal del problema y de lo que se comprende, se establece la siguiente relación:

Es una ecuación lineal homogénea, por lo cual nos remitiremos a la aplicación de la analogía de solución de los problemas que ya hemos resuelto. De esta ecuación vemos cuales son las raíces de la ecuación característica:

$$
\lambda^{2}+2 \lambda+1=0
$$

Donde $\lambda_{1}=\lambda_{2}=-1$

$Y$ la solución va a ser: $y=\left(C_{1} x+C_{2}\right) e^{-x}$. Escogemos un lenguaje apropiado para expresar la solución: la función que es solución de la ecuación diferencial dada es $y=\left(C_{1} x+C_{2}\right) e^{-x}$. (Acción 3).

- Nos preguntamos si puede ser generalizado el método de solución: Al parecer si un problema tiene características similares, tentativamente podríamos aplicar la vía de solución. 


\section{Ejemplo 1.3}

Resuelve la siguiente ecuación diferencial de segundo orden

$$
x^{2} y^{\prime \prime}+x y^{\prime}-y=0
$$

(Acción 1).

- Nos preguntamos cuáles son las expresiones de mayor valor semántico: función.

- Establecemos la incógnita: Una función $f(x)$ que satisface las condiciones que exige el problema.

- Enunciamos el problema con nuestras propias palabras: Buscamos una función derivable respecto a $x$ que cumpla la propiedad que

$$
x^{2} y^{\prime \prime}+x y^{\prime}-y=0
$$

- Parece que los datos son suficientes.

- Tampoco parece haber datos sobrantes.

- relacionamos los datos con la incógnita: relacionamos la estructura.

- transformamos el problema en uno equivalente: el problema lo transformamos a resolver una ecuación diferencial, que cumpla las propiedades anteriores.

- El problema se ubica en el campo del análisis.

- Lo clasificamos: Le damos la clasificación de reductivo por principio de reducción.

(Acción 2).

- Recordamos si ya hemos resuelto algún problema parecido o relacionado. Suponiendo que es nuevo nos damos a la tarea de crear una estrategia de solución.

- A partir de la expresión del problema y de lo que entendemos, nos trazaremos el objetivo de convertir la ecuación en alguna que ya conozcamos su solución: Aquí se idearon dos formas para desarrollar la solución al problema.

Primera forma: Hacemos la sustitución $x=e^{t}$, derivando en ambos miembros se obtiene $d x=e^{t} d t, \quad \frac{d x}{d t}=e^{t}$.

Como $y^{\prime}=\frac{d y}{d x}$, podemos dividir por $d t$. Así tenemos $y^{\prime}=\frac{\frac{d y}{d t}}{\frac{d x}{d t}}=e^{-t} \frac{d y}{d t}$.

Lo mismo hacemos para $y^{\prime \prime}$ :

$$
y^{\prime \prime}=\frac{d y^{\prime}}{d x}=\frac{\frac{d y^{\prime}}{d t}}{\frac{d x}{d t}}=\frac{e^{-t \frac{d^{2} y}{d t^{2}}-e^{-t} \frac{d y}{d t}}}{e^{t}}=e^{-2 t}\left(\frac{d^{2} y}{d t^{2}}-\frac{d y}{d t}\right) .
$$

Ahora se sustituye $y^{\prime}$ e $y^{\prime \prime}$, así como el valor de $x$ en la ecuación original y se obtiene

$$
e^{2 t} e^{-2 t}\left(\frac{d^{2} y}{d t^{2}}-\frac{d y}{d t}+e^{t} e^{-t} \frac{d y}{d t}-y=0\right)
$$




$$
\frac{d^{2} y}{d t^{2}}-y=0
$$

La cual es una ecuación homogénea y las raíces de su ecuación característica son $\mu_{1}=1, \quad \mu_{2}=-1$. Así, $y=C_{1} e^{t}+C_{2} e^{t}$ y por lo tanto $y=C_{1} x+C_{2} \frac{1}{x}$ es la solución de la ecuación diferencial dada.

Segunda forma: Hacemos $y=x^{m}$, entonces $y^{\prime}=m x^{m-1}, \quad y^{\prime \prime}=m(m-1) x^{m-2}$, al sustituir en la ecuación original y simplificar, se obtiene la ecuación $x^{m}\left(m^{2}-1\right)=0$ y las soluciones $m=1, m=-1$

Entonces $y=x, y=\frac{1}{x}$. Al ser soluciones linealmente independientes, podemos establecer la solución general como una combinación lineal de ambas: $y=C_{1} x+C_{2} \frac{1}{x}$.

(Acción 3).

- Escogemos un lenguaje apropiado para expresar la solución: La solución a la ecuación diferencial $x^{2} y^{\prime \prime}+x y^{\prime}-y=0$ es $y=C_{1} x+C_{2} \frac{1}{x}$.

- Nos preguntamos si puede ser generalizado el método de solución: Al parecer si un problema tiene características similares, tentativamente podríamos aplicar la vía de solución.

- Las dos vías de solución nos dan distintas características heurísticas, la primera de transformar la ecuación en homogénea (principio de reducción) y la segunda fue establecer una solución tentativa(método analítico).

- ¿Responde realmente al problema? Sí, porque la función determinada satisface la ecuación diferencial.

\subsection{Conclusiones}

La concepción del término "problema" que se asumió en la investigación y los elementos de la teoría de la actividad, permitieron estructurar la metodología para los procedimientos de solución de problemas sobre ecuaciones diferenciales. Ya que las actividades indicadas en cada una de las acciones, se favorecieron a partir de indentificar la función de cada una de las etapas: orientación, ejecución y control en dichos procedimientos de solución.

En cada procedimiento de solución, no sólo se mostró la potencialialidad de la heurística, sino que se fueron identificando los dominios sobre el contenido matemático que estuvo en juego.

La metodología que se propone está orientada tanto al profesor como al alumno del nivel universitario, con su aplicación a la resolución de problemas sobre ecuaciones diferenciales se favorece el proceso de enseñanza y aprendizaje de éstas. 


\section{Bibliografía}

[1] Alvero, F. Diccionario Cervantes. Editorial Pueblo y Educación (1980). La Habana.

[2] Bertoglia, L. Psicología del Aprendizaje. Universidad de Antofagasta (1990). Chile.

[3] Borasi, R. "On the nature of problems". Educational Studies of Mathematics, 17,(1986): 125-141

[4] Campitrous, L y Rizo, C. Aprende a resolver problemas aritméticos. Editorial Pueblo y Educación (1996). Cuba.

[5] Diccionaro Aristos de la Lengua Española. Editorial Pueblo y Educación (1978). Cuba.

[6] González, M. Matemática. Complementos de Aritmética y Algebra. Editorial Pueblo y Educaciń (1954). La Habana.

[7] Hadamard, J. An essay on the psychology of invention in the mathematical field. Ed Princenton University Press (1945). Princenton.

[8] Kantowski, M. G. "Mathematics Educations Research Implications for the 80". Problem Solving (Estados unidos), 4 (1981) : 111-126.

[9] Labarrere, A. F. Bases Psicológicas de la enseñanza de la resolución de problemas matemáticos en la escuela primaria. Pueblo y Educación. (1988). La Habana, Cuba.

[10] Lee, S. La heurística en la solución de ecuaciones diferenciales. Tesis de Maestría no publicada. Universidad Autónoma de Guerrero, (2009). México.

[11] Leontiev, N., Actividad, Conciencia, Personalidad. Editorial Pueblo y Educación (1976). La Habana.

[12] Leontiev, N. Sobre la Formación de Capacidades. Editorial Pueblo y Educación (1986). La Habana.

[13] Majmutov, M., Enseñanza Problémica. Editorial Pueblo y Educación (1983). La Habana.

[14] Palacios, C. y Zambrano, E., "Aprender y enseñar ciencias: una relación a tener en cuenta". En Proyecto Principal de Educación en América Latina y el Caribe. Bol 31 (1993) UNESCO. Santiago de Chile.

[15] Polya, G. How to solve it. Princenton University press (1945). USA

[16] Polya, G. Mathematical Discovery: On Understanding, Learning and Teaching Problem Solving. Combined edition (1981). New York, USA.

[17] Rodríguez, A. "Un esquema para la solución de problemas en Matemáticas". Boletín SCMC 13, (1991): 10-20.

[18] Rubinstein, S. L. El proceso de pensamiento. Editora Nacional de Cuba (1966). La Habana.

[19] Schoenfeld, A. H. Learning to think mathematically. Problem Solving, metacognition and sense making in mathematics. University of California. (1991). California, USA.

[20] Sigarreta J. M. y Arias L. R. "La resolución de problemas: Un recurso para el desarrollo de la formación de la personalidad". Revista de la Sociedad Argentina de Educación Matemática 20, 13-22. (2003).

[21] Sigarreta J. M. y Laborde J. M. "Estrategias para la Resolución de Problemas como recurso para la interacción sociocultural". Revista de la Sociedad Argentina de Educación Matemática 20, 17-29. (2004).

[22] Sigarreta J. M. y Palacio J. "Mathematic problems feature in order to develop values education". Ciencias Holguín 8(3). (2001). Obtenido en Marzo 15, 2009, de http: //www.ciencias.holguin.cu/ 2001/Diciembre/articulos/ARTI5.HTM

[23] Sigarreta J. M. y Torres. J. "Utilización de los problemas Matemáticos en la formación de valores". EMA 8, 35-45. (2003). 
[24] Sigarreta J. M. and Ruesga P. "Estrategia para la Resolución de Problemas, utilizando las Funciones". Docencia Universitaria 5 (1-2), 75-95. (2004).

[25] Sigarreta J. M., Ruesga P. y Rodríguez J. M. (2006). "The problem solving: A historical-didactical vision". Boletín de la Asociación Matemática Venezolana 13(1), 53- 67. (2004).

[26] Sigarreta J. M., Ruesga P. y Rodríguez J. M. "Analogies in Differential Equations". Far East Journal of Mathematical Education 2(1), 31-48. (2008).

[27] Sigarreta, J. M. y Palacio, J. "La resolución de problemas matemáticos y su incidencia en la formación de personalidad". (2001). En Evento internacional de Matemática e Informática MATINFO. Universidad de Holguín, Cuba.

[28] Zillmer, W., Complementos de Metodología de la Enseñanza de la Matemática. Editorial Pueblo y Educación (1981). La Habana. 